\title{
Lateral Subtalar Dislocation of the Foot: A case report
}

\author{
by J. Terrence Jose Jerome, MBBS, DNB (Ortho), MNAMS (Ortho) ${ }^{1} \rrbracket$, Mathew Varghese, M.S. \\ $\left(\right.$ Ortho $^{2}{ }^{-}$, Balu Sankaran, FRCS, FAMS ${ }^{3}$, K. Thirumagal, MD $^{4}$
}

The Foot \& Ankle Journal 1 (12): 2

Subtalar dislocation is the simultaneous dislocation of the distal articulations of the talus at both the talocalcaneal and talonavicular joints. It can occur in any direction and always produce significant deformity. Most common is the medial dislocation. Less common presentations are lateral, anterior and posterior dislocations. These dislocations are associated with osteochondral fractures. Closed reduction and immobilization remains the mainstay of treatment. Radiographs and computed tomography scan confirms the post reduction alignment stability of subtalar joints and intra-articular fracture fragments. We report a case of lateral subtalar dislocation without osteochondral fracture fragments in a 30-yearold man.

Key words: Subtalar dislocation, dislocated talus, closed reduction, immobilization

This is an Open Access article distributed under the terms of the Creative Commons Attribution License. It permits unrestricted use, distribution, and reproduction in any medium, provided the original work is properly cited. (The Foot \& Ankle Journal (www.faoj.org)

Subtalar dislocations are rarely found in routine orthopedic practice. Many of these dislocations result from high-energy injuries such as falls from a height, athletic injuries or a motor vehicle accident. ${ }^{1}$ Inversion or eversion force is dissipated through the weak talonavicular and talocalcaneal ligaments, which eventually result in subtalar dislocation.

There are two types of subtalar dislocation. In lateral subtalar dislocation, the head of talus is found medially and the rest of the foot is dislocated laterally. In medial subtalar dislocation, the head of the talus is found laterally and the rest of the foot is dislocated medially.

Address correspondence to: Dr. J. Terrence Jose Jerome, MBBS.,DNB (Ortho), MNAMS (Ortho)

Registrar in Orthopedics, Dept. of Orthopedics

St. Stephen's Hospital, Tiz Hazari, Delhi 54, India

\footnotetext{
${ }^{1}$ Registrar in Orthopedics, Department of Orthopedics, St. Stephens Hospital, Tiz Hazari, Delhi, India.

${ }^{2}$ Head Professor, Department of Orthopedics, St. Stephens Hospital, Tiz

Hazari, Delhi, India.

${ }^{3}$ Professor Emeritus, Orthopedics, St. Stephens Hospital, Tiz Hazari, Delhi, India. E-mail: pasle@bol.net.in

${ }^{4}$ Professor, Orthopedics, Tamilu, India
}

Medial dislocation has been referred to as an "acquired clubfoot", while the lateral injury is described as an "acquired flatfoot". We present a case of an adult with lateral subtalar dislocation following a fall.

\section{Case Report}

A 30-year-old man who sustained a fall from the stairs came to our emergency department with pain and swelling of the right foot. The foot was diffusely swollen with minimal laceration and tenting of the skin over the prominent talar head which was felt medially. The rest of the foot was found dislocated laterally. (Figs. 1 and 2) Pulse of the posterior tibial and dorsalis pedis artery were not felt due to massive soft tissue distortion. Radiograph of the right foot showed lateral subtalar dislocation. (Figs. 3 and 4) Doppler ultrasound showed normal arterial flow in both posterior tibial and dorsal pedis arteries. 


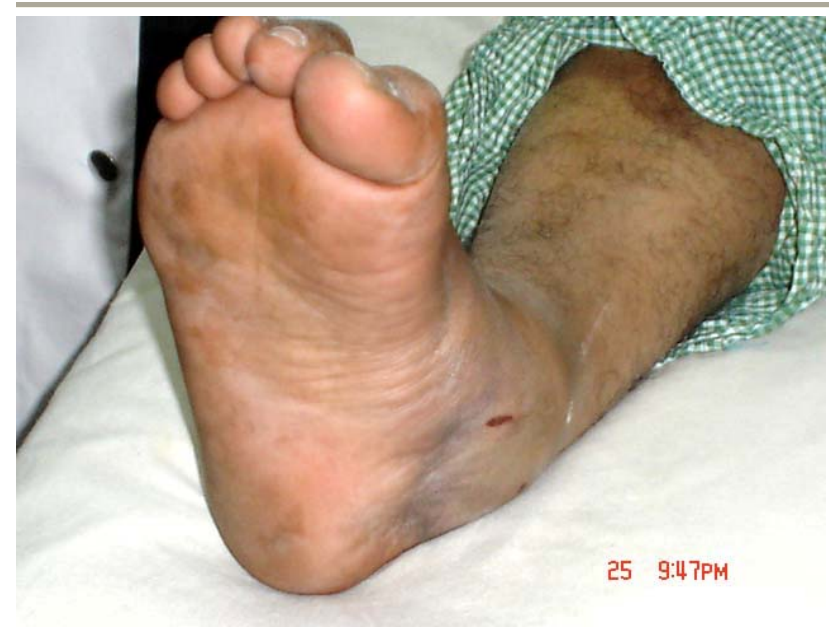

Figure 1 The foot was diffusely swollen with minimal laceration and tenting of the skin over the prominent talar head which was felt medially.

Closed reduction was done under spinal anesthesia. Firm manual foot traction with counter-traction on the leg combined with direct digital pressure over the head of talus aided in smooth reduction, which was associated with an audible clunk.

Post reduction radiographs showed normal and stable alignment of subtalar and talo-navicular joints without osteochondral fractures. (Figs. 5 and 6)

Computer tomography (CT) scan confirmed the absence of osteochondral fractures and the stability of the subtalar joints. The patient was immobilized in a short-leg posterior plaster splint for 4 weeks. Following immobilization, the patient underwent a vigorous, active exercise program. The patient progressed to weight bearing and active range of motion exercises to regain subtalar and midtarsal joint motion. Two years after the injury, the patient had a stable, relatively good functional foot with minimal pain on walking on uneven ground.

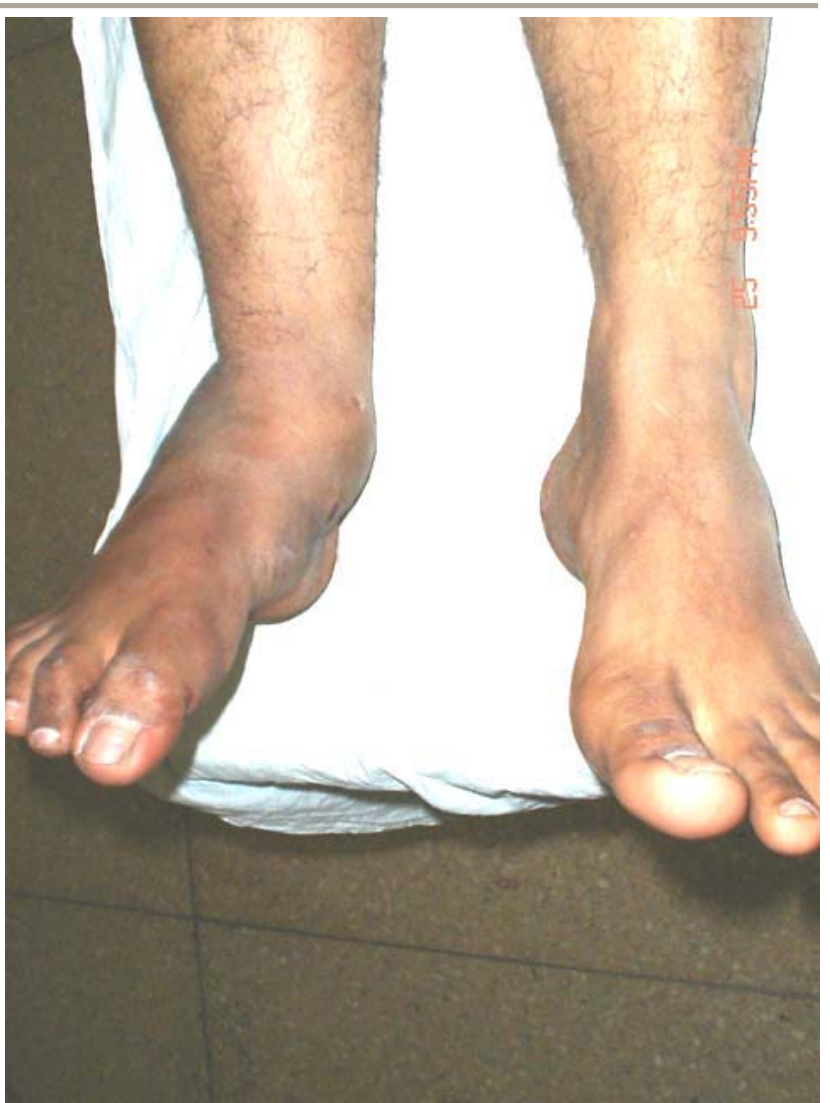

Figure 2 The right foot was found to be dislocated laterally.

\section{Discussion}

Subtalar dislocation by definition has a normal tibiotalar joint. Most dislocations occur in males (6:1) of early age. Subtalar dislocation can occur in any direction and always produce significant deformity.

Most commonly (80\% to $85 \%$ ), the foot is displaced medially with the calcaneus lying medially, the head of the talus prominent dorsolaterally, and the navicular medial and sometimes dorsal to the talar head and neck. ${ }^{1,2,3}$ Less commonly (15\% to $20 \%$ ), lateral dislocation occurs. 


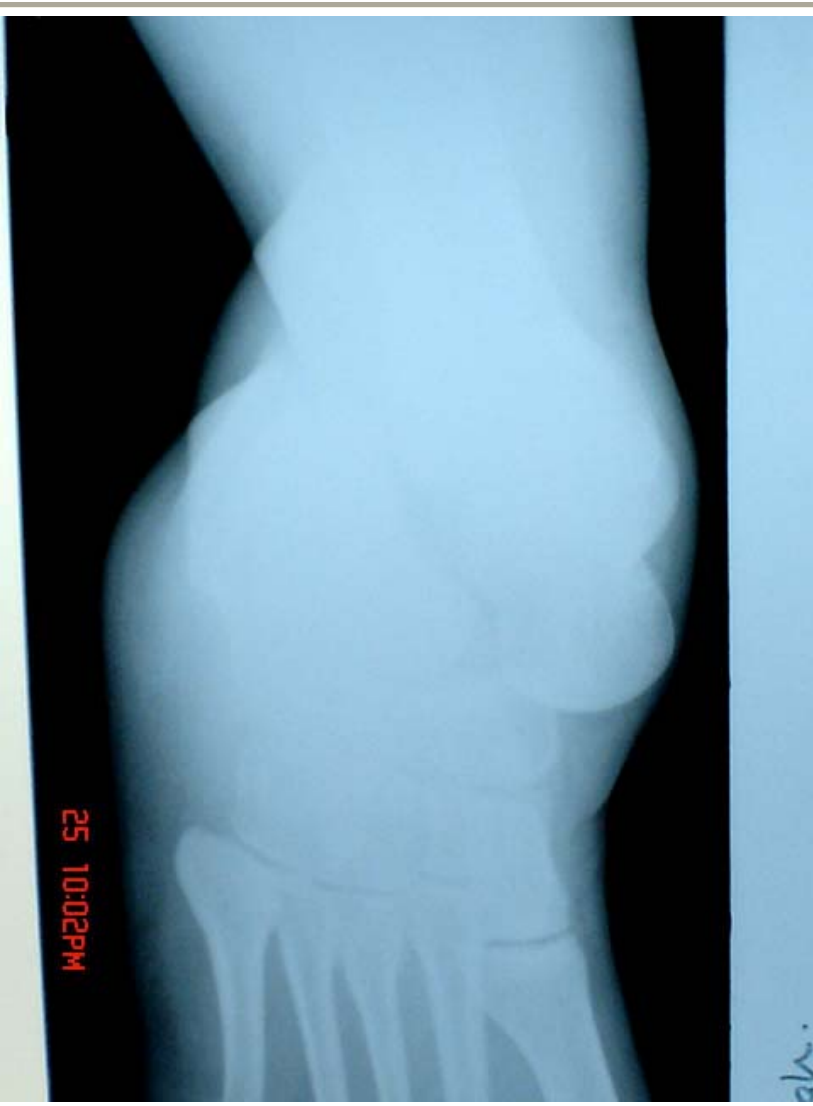

Figure 3 The dorsoplantar view shows the dislocation of the talo-navicular and subtalar joints. Head of the talus was seen lying medially. Normal alignment of calcaneo-cuboid joint is appreciated.

Inversion of the foot results in a medial subtalar dislocation, while eversion produces a lateral dislocation. The strong calcaneonavicular ligament resists disruption, and the inversion or eversion force is dissipated through the weaker taloavicular and talocalcaneal ligaments. This disrupts these two joints which causes displacement of the calcaneus, navicular and all distal bones of the foot as a unit, either medially or laterally. ${ }^{2,3}$

The sustentaculum tali acts as a fulcrum about which the foot rotates to lever apart the talus and calcaneus in medial subtalar dislocation. The foot pivots about the anterior process of the calcaneus, again causing the talus and calcaneus to separate in lateral subtalar dislocation. , $^{1,2,4}$

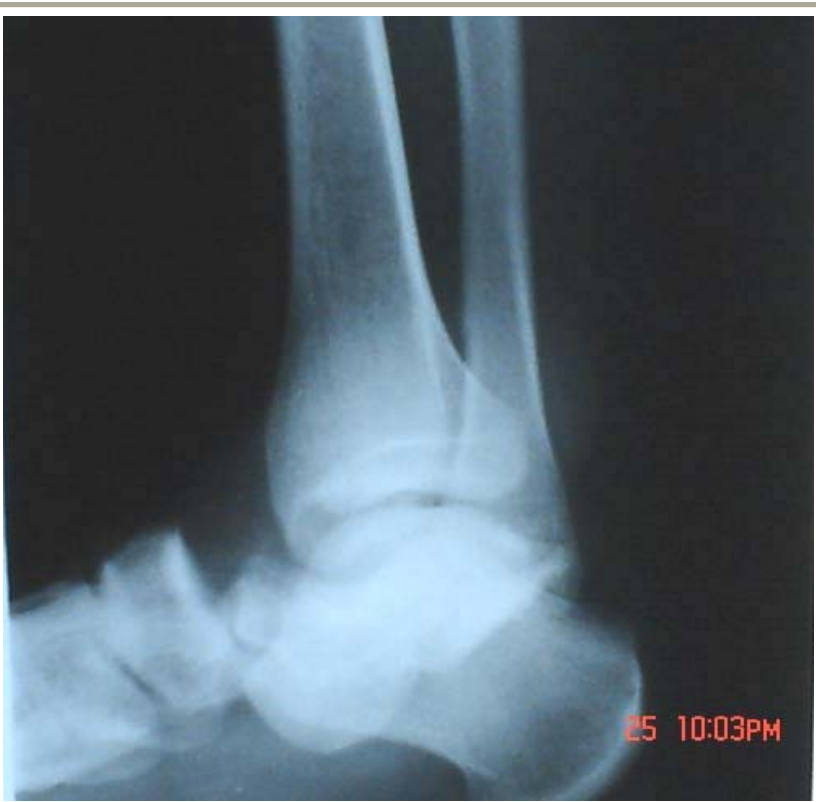

Figure 4 The lateral view again shows the dislocation of the talo-navicular and subtalar joints. Head of the talus is severely declinated.

Rare cases of anterior ${ }^{5}$ and posterior ${ }^{1}$ displacement of the foot after subtalar dislocation have also been reported. It is important to distinguish the medial or lateral subtalar dislocations because the method of reduction is different and the long-term prognosis appears to be worse with the lateral dislocation.

Between $10 \%$ and $40 \%$ of subtalar dislocations are open. ${ }^{6}$ Open injuries tend to occur more commonly with the lateral subtalar dislocation pattern and probably as the result of a more violent injury. ${ }^{6}$ Long term follow-up demonstrated very poor results with the open subtalar dislocations.

The keystone of treatment for all subtalar dislocations is prompt and gentle reduction under general or spinal anesthesia. ${ }^{7}$ All open injuries must be thoroughly debrided at the time of reduction, and the wound should be left open, with delayed primary closure anticipated in 3 to 5 days. Due to the high incidence of associated articular fracture and associate poor prognosis, CT scan of the foot and ankle should be obtained after reduction and splinting.

(C) The Foot \& Ankle Journal, 2008 


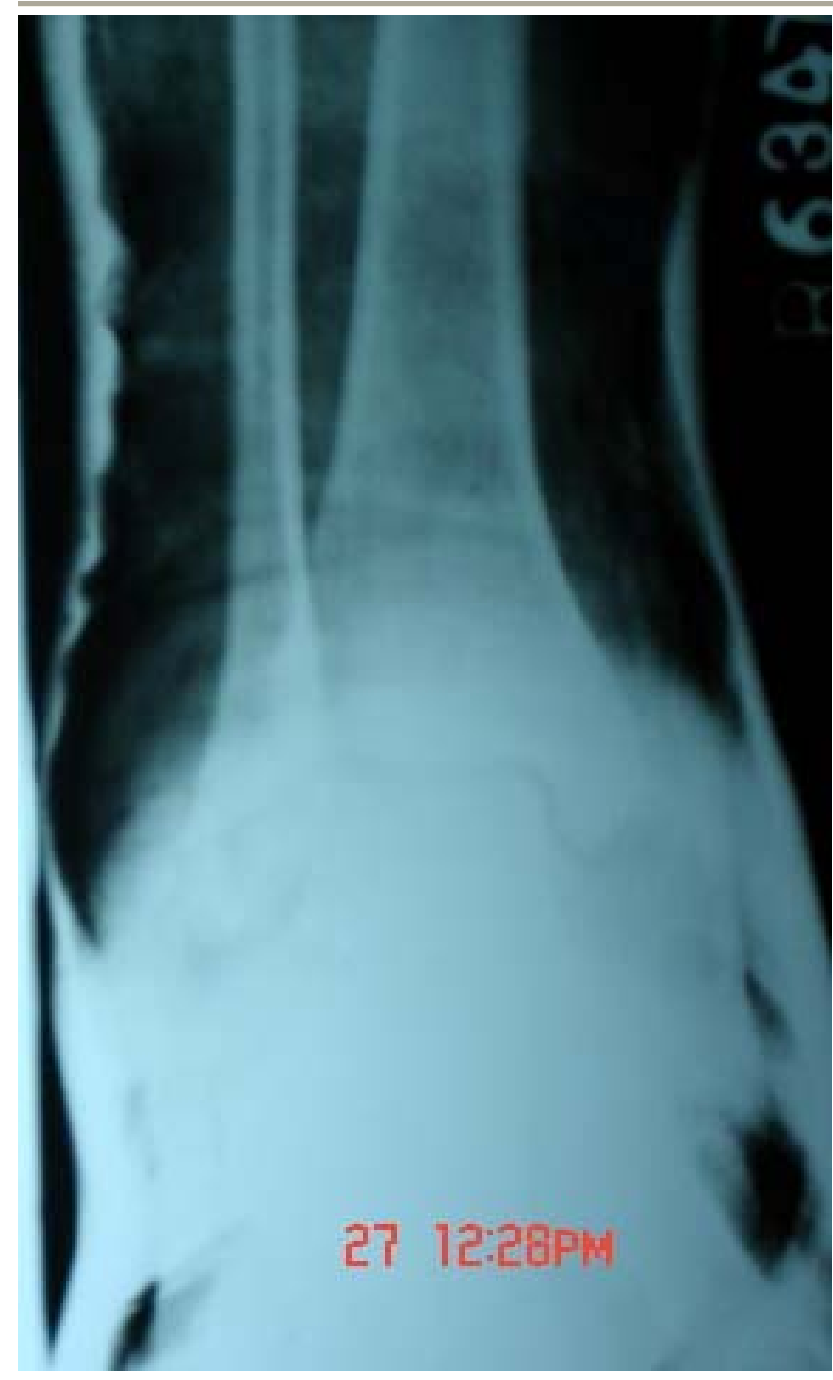

Figure 5 The post reduction anterior posterior radiograph showed normal and stable alignment of subtalar and talo-navicular joints without osteochondral fractures.

Simple dislocation that is reduced readily by closed reduction and do not have associated fracture, do very well. ${ }^{1}$ In approximately $10 \%$ of medial subtalar dislocations and $15 \%$ to $20 \%$ of lateral dislocations, closed reduction cannot be achieved. ${ }^{3,8}$ Soft tissue interposition and bony blocks have been identified as factors preventing closed reduction. Another common obstruction to closed reduction in medial dislocations is an impaction fracture of the articular surface of talus and navicluar. ${ }^{7}$

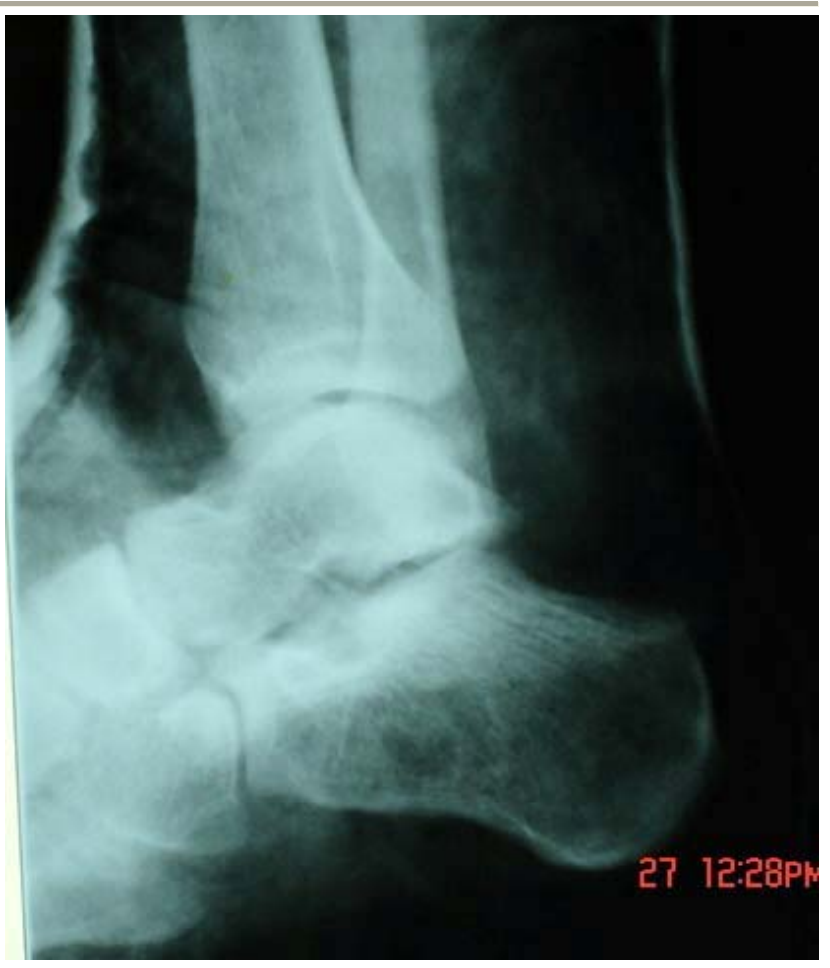

Figure 6 The post reduction lateral radiograph showed normal and stable alignment of subtalar and talo-navicular joints without osteochondral fractures.

In comparison, the most common obstruction to closed reduction in lateral subtalar dislocation is the interposed tibialis posterior tendon. ${ }^{8}$

Open reduction is done for irreducible medial, lateral subtalar dislocations and osteochondral fracture fragments which blocks closed reduction. Any small, loose articular fracture fragments should be removed. Large intra-articular fractures should be reduced and fixed with Kirschner wires or small screws to restore joint stability and congruity. ${ }^{9}$

The only consistent complication in simple uncomplicated dislocations is limitation of subtalar joint motion, with the occasional associated symptoms of difficulty in walking on uneven ground and pain in the foot with weather changes. $^{2,7}$ 
Lancaster and co-workers noted a poorer prognosis when there were associated injuries such as soft tissue injury, open contaminated injuries, extra-articular fracture, intra-articular fracture, infections, lateral subtalar dislocations, neglected subtalar dislocations and osteonecrosis.

Our patient, who had sustained a fall from stairs, came with a diffusely swollen foot with the head of talus felt medially and the rest of the foot dislocated laterally as a unit. Radiographs confirmed the lateral subtalar dislocation. There was no associated osteochondral fracture. Simple closed reduction was successful. Our literature review showed few reports of isolated lateral subtalar dislocation.

We emphasize the importance of proper diagnosis and timely management of dislocations around the subtalar joint, as these tend to result in a significant deformity with joint stiffness. Lateral subtalar dislocation is one such type dislocation which is not mentioned in the literature and should be carefully treated. There should always be a high index of suspicion concerning associated osteochondral fractures. CT scan should be performed after reduction to assess for the intra-articular fractures of the subtalar joint. Open reduction is recommended for irreducible dislocations and fixation is recommended in large displaced, articular fractures that can produce subtalar joint incongruity.

\section{References}

1. DeLee JC, Curtis R. Subtalar dislocation of the foot. J Bone Joint Surg 64A: 433 - 437, 1982.

2. Grantham SA. Medial Subtalar dislocation: five cases with a common etiology. J Trauma 4 (11): 845 - 849, 1964.

3. Heppenstall RB, Farahvar H, Balderston R, Lotke P. Evaluation and management of subtalar dislocations. J Trauma 20 (6): 494 - 497, 1980.

4. Monson ST, Ryan JR. Subtalar dislocation. J Bone Joint Surg 63A (7): $1156-1158,1981$.

5. Inokuchi S, Hashimoto T, Usami N. Anterior subtalar dislocation: case report. J Orthop Trauma 11(3): 235 - 237, 1997.

6. Goldner JL, Poletti SC, Gates HS 3rd, Richardson WJ. Severe open subtalar dislocations: long-term results. J Bone Joint Surg 77A (7):1075 - 1079, 1995.

7. Bohay DR, Manoli A $2^{\text {nd }}$. Subtalar dislocations. Foot Ankle Int 16(12): 803 - 808, 1995.

8. Leitner B. Obstacles to reduction in subtalar dislocations. J Bone Joint Surg 36A (2): 299 - 306, 1954.

9. Naranja RA Jr, Monaghan BA, Okereke E, Williams GR Jr. Open medial subtalar dislocation associated with posterior process fracture of the talus. J Orthop Trauma 10(2): 142 - 144, 1996.

10. Lancaster S, Horowitz M, Alonso J. Subtalar dislocations: a prognosticating classification. Orthopedics 8 (10): 1234-1240, 1985. 